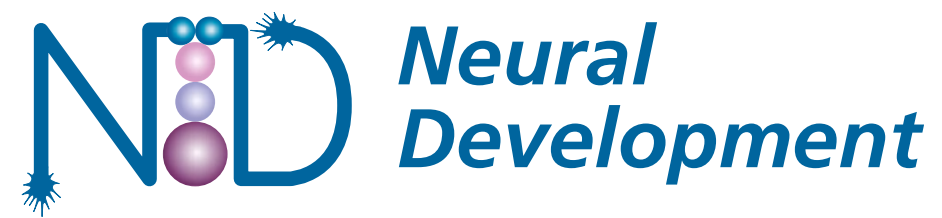

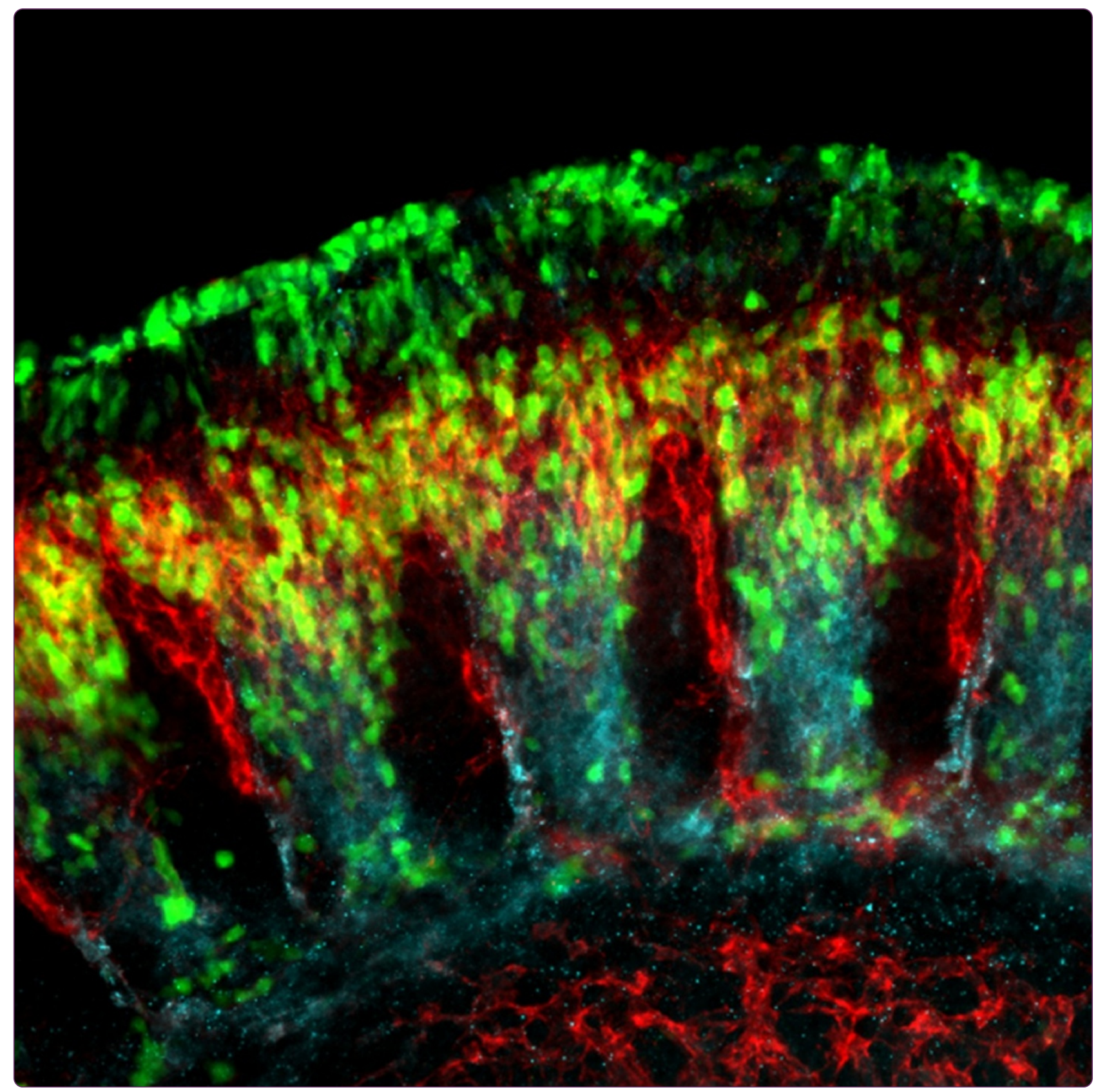

\title{
Neuropilins define distinct populations of neural crest cells
}

Lumb et al.

C Biomed Central 


\title{
Neuropilins define distinct populations of neural crest cells
}

\author{
Rachael Lumb ${ }^{1,2}$, Sophie Wiszniak', Samuela Kabbara ${ }^{1}$, Michaela Scherer ${ }^{1}$, Natasha Harvey $^{1}$ and Quenten Schwarz ${ }^{1 *}$
}

\begin{abstract}
Background: Neural crest cells (NCCs) are a transient embryonic cell type that give rise to a wide spectrum of derivatives, including neurons and glia of the sensory and autonomic nervous system, melanocytes and connective tissues in the head. Lineage-tracing and functional studies have shown that trunk NCCs migrate along two distinct paths that correlate with different developmental fates. Thus, NCCs migrating ventrally through the anterior somite form sympathetic and sensory ganglia, whereas NCCs migrating dorsolaterally form melanocytes. Although the mechanisms promoting migration along the dorsolateral path are well defined, the molecules providing positional identity to sympathetic and sensory-fated NCCs that migrate along the same ventral path are ill defined. Neuropilins (Nrp1 and Nrp2) are transmembrane glycoproteins that are essential for NCC migration. Nrp1 and Nrp2 knockout mice have disparate phenotypes, suggesting that these receptors may play a role in sorting NCCs biased towards sensory and sympathetic fates to appropriate locations.

Results: Here we have combined in situ hybridisation, immunohistochemistry and lineage-tracing analyses to demonstrate that neuropilins are expressed in a non-overlapping pattern within NCCs. Whereas Nrp1 is expressed in NCCs emigrating from hindbrain rhombomere 4 ( $r 4$ ) and within trunk NCCs giving rise to sympathetic and sensory ganglia, Nrp2 is preferentially expressed in NCCs emigrating from $r 2$ and in trunk NCCs giving rise to sensory ganglia. By generating a tamoxifen-inducible lineage-tracing system, we further demonstrate that Nrp2-expressing NCCs specifically populate sensory ganglia including the trigeminal ganglia $(V)$ in the head and the dorsal root ganglia in the trunk.

Conclusions: Taken together, our results demonstrate that Nrp1 and Nrp2 are expressed in different populations of NCCs, and that Nrp2-expressing NCCs are strongly biased towards a sensory fate. In the trunk, Nrp2-expressing NCCs specifically give rise to sensory ganglia, whereas Nrp1-expressing NCCs likely give rise to both sensory and sympathetic ganglia. Our findings therefore suggest that neuropilins play an essential role in coordinating NCC migration with fate specification.
\end{abstract}

Keywords: Neural crest cell, Neuropilin, Sensory neuron, Sympathetic neuron

\section{Background}

Neural crest cells (NCCs) are transient embryonic cells that generate a wide spectrum of derivatives. Based on their origin along the anteroposterior axis, NCCs are broadly divided into different populations that give rise to a restricted set of derivatives. Cranial NCCs arise anterior to the fifth somite, and form derivates such as sensory, sympathetic, and parasympathetic ganglia, connective tissue, bone, cartilage, and muscle tendons of the head [1].

\footnotetext{
* Correspondence: quenten.schwarz@health.sa.gov.au

${ }^{1}$ Centre for Cancer Biology, University of South Australia and SA Pathology,

Frome Road, Adelaide 5000, Australia

Full list of author information is available at the end of the article
}

Trunk NCCs arise posterior to the fourth somite, and give rise to cell types such as sympathetic and sensory neurons, adrenal chromaffin cells, and melanocytes [2]. Vagal NCCs arise from the level of somites 1 to 7 , and produce enteric NCCs that form the neurons and glia of the enteric nervous system [3], and other cell types such as cardiac NCCs (level of somites 1 to 3 ) that form vascular smooth muscle lining the great arteries and contribute to the aorticpulmonary septum [4].

Even within these anatomically defined domains, NCCs can be further subdivided by additional properties, including their migration path and their developmental fate [5-7]. For example, cranial NCCs migrate in a segmented 
pattern to populate specific regions of the face and neck. This segmentation is critical for the correct formation and positioning of cranial NCC derivatives, and is under the control of cell intrinsic guidance receptors and signals provided by the environment in which NCCs travel and ultimately differentiate $[8,9]$. Trunk NCCs also migrate along distinct paths that correlate with their developmental fate [5]. Thus, NCCs migrate ventrally, and either stop within the sclerotome to give rise to sensory neurons and glia within the dorsal root ganglia (DRG), or migrate towards the dorsal aorta to give rise to the neurons and glia of the sympathetic nervous system. A proportion of these ventrally migrating NCCs that initially migrate along axonal pathways also give rise to melanocytes [10]. By contrast, NCCs migrating dorsolaterally between the dermomyotome and epidermis give rise only to melanocytes. A fundamental question for this field is how NCCs navigate their environment to position themselves in appropriate locations.

There is mounting evidence from chick and mouse studies supporting the notion that NCCs migrating dorsolaterally are specified at the time of delamination $[6,11-13]$, and that lineage-specific expression of cell surface receptors promotes migration along this path [5]. Indeed, several receptor-ligand pairs, including the Slit/ ROBO [14], Eph/ephrin [11], endothelin/EDNR [11], and Steel factor/KIT $[15,16]$ families have been implicated in permitting access to the dorsolateral path. By contrast, there are two competing models to explain the timing at which ventrally migrating NCCs become specified towards the sensory or sympathetic lineages. On the one hand, it is proposed that this population of NCCs are specified prior to or at the time of delamination, and that lineage-specific properties promote migration along restricted pathways. This notion is derived from the findings that neurogenin 2 (Ngn2)-expressing NCCs are strongly biased towards a sensory fate in mice [17], and that nociceptive neurons arise specifically from contralaterally migrating NCCs within the chick neural tube [18]. Specification of pre-migratory NCCs is also supported by lineage-tracing experiments detailing a spatiotemporal fate map of sympathetic- and sensory-fated cells within the chick dorsal neural tube [19]. However, using in vivo optical imaging to analyse the behaviour of single cells, McKinney and colleagues recently found that pre-migratory NCCs in the chick lack positional identity or fate prior to delamination [20], but rather, they migrate from the neural tube stochastically and rapidly change their expression profiles as they migrate. This and other lineage/transplantation studies in chick support an alternative model in which migrating NCCs are proposed to be multipotent, becoming specified by their environment only as they migrate towards or at their target regions [21-23].
The molecular cues that cause some ventrally migrating NCCs to remain near the neural tube to form sensory ganglia, while others continue migrating to adopt other fates (e.g. sympathetic ganglia) remain ill defined. In chick, the chemokine receptor CXCR4 is expressed in a specific subset of early migrating NCCs to control their migration towards a source of SDF1 around the anlagen of the sympathetic ganglia [24,25]. Neuropilins (Nrp1 and Nrp2) are transmembrane receptors for guidance molecules of the class 3 semaphorin (SEMA3) family and for heparin-binding isoforms of vascular endothelial growth factor (VEGF) [26]. Nrp1 and Nrp2 are expressed in both cranial NCCs and ventrally migrating trunk NCCs, and are required for proper segmentation of the peripheral nervous system [27-30]. Notably, phenotypic analysis of single Nrp1 or Nrp2 knockout embryos identified distinct migration defects in different NCC lineages. In the head, NCCs emigrating from $\mathrm{r} 4$ are misguided in Nrp1 knockout embryos [31], while NCCs emigrating from r2 are misguided in Nrp2 knockout embryos [31,32]. Accordingly, neurons arising from these populations of cranial NCCs are ectopically positioned within neuropilin knockout embryos: neurons from the facio-acoustic ganglia (VII-VIII) are ectopically positioned in Nrp1 knockout embryos, while neurons from the trigeminal ganglia (V) are ectopically positioned in Nrp2 knockout embryos $[31,32]$. These lineage-specific NCC defects also extend to the trunk, where Nrp1 knockout embryos have ectopically placed neurons in the sympathetic and sensory nervous systems, and Nrp2 knockout embryos have misplaced neurons in the sensory nervous system [28,33,34]. In combination with expression analyses, these observations suggest that neuropilins may be required for the migration of distinct populations of NCCs, and that this may be controlled by the restricted expression of Nrp1 and Nrp2 in NCCs biased towards sympathetic and/or sensory fates [28]. However, to date, this notion has lacked definitive support from co-expression analyses and fate mapping of neuropilin-expressing NCCs.

Here we have combined in situ hybridisation, immunohistochemistry and lineage-tracing analyses to directly compare the expression of Nrp1 and Nrp2 in migrating NCCs. Our results demonstrate that these receptors are expressed in a non-overlapping pattern within different populations of cranial and trunk NCCs. Whereas Nrp1 is expressed in cranial NCCs migrating out of hindbrain r4 and within trunk NCCs migrating towards the sympathetic and sensory ganglia, Nrp2 is preferentially expressed in cranial NCCs migrating out of r2 and in trunk NCCs migrating towards and positioned within the sensory ganglia. To determine whether Nrp2-expressing NCCs are fate-restricted towards the sensory lineage, we generated a tamoxifen-inducible Cre/LoxP tracing system to genetically label and lineage-trace this population of cells. In 
combination with our detailed expression analyses and previous phenotypic analyses [33,34], our finding that Nrp2-expressing NCCs give rise to sensory neurons in the DRG and not the sympathetic chain identifies an essential role for neuropilins in coordinating NCC migration with fate specification.

\section{Results and discussion}

Nrp1 and Nrp2 are expressed in different populations of cranial NCCs

Previous studies, including our own, demonstrated that Nrp1 and Nrp2 knockout mice have non-overlapping phenotypes in different cranial NCC derivatives [31,32]. To address if these receptors may be expressed in distinct populations of cranial NCCs, and thereby explain the distinct migration phenotypes of neuropilin knockout embryos, we completed whole mount in situ hybridisation on wild-type embryos between embryonic day (E) 8.5 to E9.5. By comparing the expression profiles of Nrp1 and Nrp2 with that of the pan-NCC marker Sox10, we found that neuropilins are expressed in different populations of cranial NCCs. At E8.5 and E9.0, the time at which NCCs have delaminated from the neural tube and begun to migrate into the branchial arch tissue, the expression of Nrp1 was restricted to the stream of NCCs emigrating out of $\mathrm{r} 4$, while $\mathrm{Nrp} 2$ was expressed reciprocally within NCCs emigrating out of r2 (Figure 1A-F). At E9.5, this restricted expression pattern was maintained; however, low levels of Nrp2 could also be detected in or around the $\mathrm{r} 4$ stream of NCCs (Figure 1G-I). In addition to this presumptive staining within NCCs, Nrp1 expression was identified in the heart, gut, and arteries (Figure 1B, E, H), while Nrp2 expression was evident in the dorsal regions of the heart (Figure 1C, F, I).

To investigate whether neuropilins are expressed in NCCs and not other branchial arch tissue such as the surface epithelia or cranial mesoderm, we sectioned E8.5 embryos transversely through the $\mathrm{r} 2$ and $\mathrm{r} 4$ regions, and counterstained with antibodies specific to the neurotrophin receptor p75. Sox 10 and p75 were co-expressed in all migrating cranial NCCs at this stage of development (Figure 1J-M). Consistent with the results of whole mount staining, $\mathrm{Nrp} 1$ expression was identified specifically in NCCs emigrating out of $\mathrm{r} 4$ and also within non-NCC tissues such as the cranial mesoderm and the developing heart (Figure 1N, P). Conversely, Nrp2 expression was restricted to NCCs emigrating out of $\mathrm{r} 2$ and additional tissues such as the cranial mesoderm, neural tube, and bulbus cordis region of the developing heart (Figure 1R, T).

To compare expression of Nrp1 and Nrp2 in NCCs, we immunostained embryos generated by crossing Wnt1Cre [35] mice with $Z / E G$ [36] reporter mice to specifically label all NCCs and their derivatives with green fluorescent protein (GFP). Whole mount immunofluorescence staining clearly demonstrated that Nrp1 and Nrp2 are expressed in a reciprocal pattern, replicating the mRNA expression seen in our previous in situ hybridisation analysis (Figure 2A-D). Immunostaining also identified expression of Nrp1 and Nrp2 in blood vessels around the r2 and $\mathrm{r} 4$ migrating NCCs (Figure 2A-D). Longitudinal sections through the head confirmed this restricted expression along the anteroposterior axis, as well as expression within the developing vasculature (Figure 2E-H). The membrane localisation of neuropilins is consistent with their role as cell surface receptors for guidance molecules of the SEMA3 and VEGF families [30]. Taken together, our in situ hybridisation, immunohistochemical, and lineagetracing analyses confirm that neuropilins are expressed in different populations of cranial NCCs that are defined by their position along the anteroposterior axis. These restricted expression profiles therefore explain the disparate phenotypes of neuropilin knockout mice in which $\mathrm{Nrp} 1$ has ectopically placed neurons of the facio-acoustic cranial ganglia (VII-VIII) and Nrp2 has ectopically placed neurons of the trigeminal ganglia (V) $[31,32]$. Future work addressing how the expression of neuropilins is controlled should provide essential clues to the mechanisms dividing cranial NCCs into distinct populations.

\section{Nrp1 and Nrp2 are expressed in different populations of trunk NCCs}

Nrp1 and Nrp2 knockout embryos also displayed disparate phenotypes in different trunk NCC derivatives [33,34]. To explore if neuropilins are also expressed in different populations of ventrally migrating trunk NCCs, we compared their expression with that of the pan-NCC markers Sox 10 and p75 over the forelimb region of E9.5 embryos. In situ hybridisation confirmed that Nrp1 was expressed within NCCs migrating in the anterior half of the somite and in non-NCC derivatives such as the dorsal aorta and intersomitic blood vessels (Figure 3B). Nrp2 was robustly expressed in the anterior half of the somite in a pattern that partially overlapped with Sox10 (Figure 3C). To determine if neuropilins are expressed within NCCs or the supporting somitic tissue, we next counterstained longitudinal in situ hybridisation sections with anti-p75 antibodies (Figure 3D-I). In addition to labelling Sox10expressing NCCs, p75 also recognised neuroepithelial cells within the neural tube (Figure 3D, G). Co-staining of Nrp1 and p75 within the anterior half of the somite indicated that Nrp1 was expressed in trunk NCCs migrating along the ventral path (Figure 3E, H). In addition, Nrp1 was also expressed in the posterior somite and within intersomitic blood vessels. Nrp2 was broadly expressed throughout the entire anterior half of the somite, with some cells costaining with p75 (Figure 3F, I).

To investigate whether neuropilins are co-expressed or differentially expressed within distinct populations of 


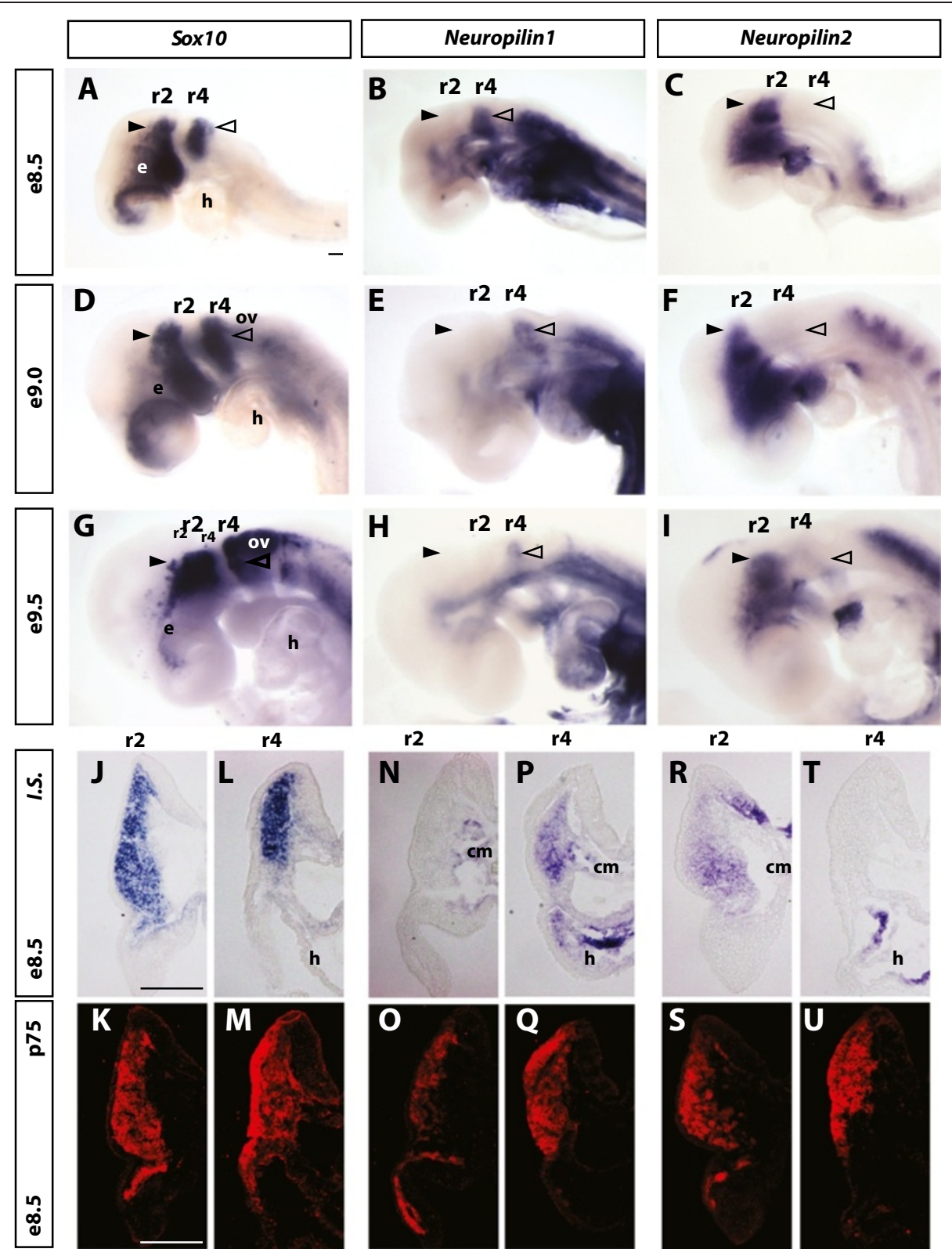

Figure 1 Nrp1 and Nrp2 are expressed in different populations of cranial NCCs. (A-I) Whole mount in situ hybridisation of E8.5 to E9.5 wild-type embryos to determine the expression profiles of Nrp1 and Nrp2. (A, D, G) Sox10 labelled all migrating cranial NCCs and defined the $r 2$ (open arrowhead), r4 (closed arrowhead) and $r 6$ populations at E8.5, E9.0, and E9.5 as well as the otic vesicle (ov) at E9.5. (B, E, H) Nrp1 was specifically expressed in the $r 4$ stream of NCCs and not in the $\mathrm{r} 2$ stream. (C, F, I) Nrp2 expression was restricted to the $\mathrm{r} 2$ stream of NCCs and was lacking in the $\mathrm{r} 4$ stream at E8.5 and E9.0. At E9.5, low levels of Nrp2 could be detected in the tissue ventral to $\mathrm{r} 4$. (J-U) Transverse in situ hybridisation sections of E8.5 embryos through the regions of the $r 2$ and $r 4$ streams counterstained for the neurotrophin receptor p75. (J-M) Sox10 and p75 identified all migrating NCCs. p75 staining was also evident in the epithelia in close association with migrating NCCs in the region ventral to r4. (N-Q) Transverse sections confirmed that Nrp1 is specifically expressed in the r4 NCC stream as well as the developing heart and cranial mesoderm (cm). (R-U) Transverse sections confirmed that Nrp2 was restricted to the 12 stream of NCCS as well as the neural tube, cranial mesoderm, and developing heart. IS, in situ hybridisation; e, eye; $h$, heart, r2, rhombomere 2; r4, rhombomere 4; ov, otic vesicle; e, eye. Scale bar = $100 \mu \mathrm{m}$.

ventrally migrating NCCs, we performed immunofluorescence analysis on E9.5 Wnt1Cre; Z/EG embryos. Whole mount staining for GFP, Nrp1 and Nrp2 identified mixed populations of NCCs expressing the neuropilin receptors (Figure 4A-E). Consistent with our in situ hybridisation analysis results, Nrp1 was identified in NCCs in the anterior half of the somite, the intersomitic blood vessels, and the dorsal aorta (Figure 4D). Nrp2 was also expressed in NCCs in the anterior half of the somite in addition to the intersomitic blood vessels. Notably, 


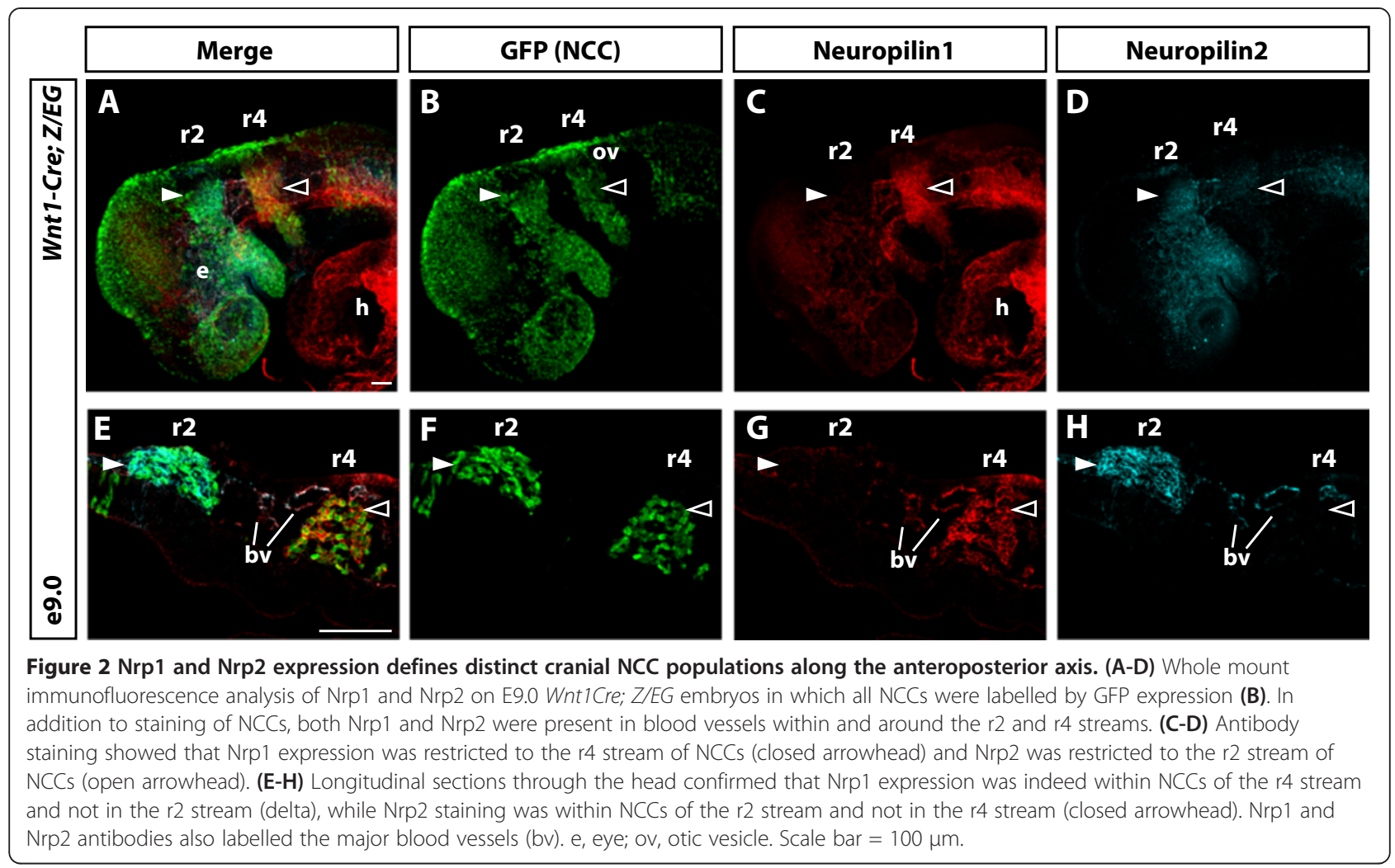

there was limited overlap of the Nrp1 and Nrp2 expression domains, and NCCs expressing high levels of Nrp1 with low or absent Nrp2 could be identified in the dorsal regions of the somite (Figure 4A-E arrow).

To define the expression of neuropilins within individual trunk NCCs, we next analysed transverse sections of E9.5 embryos taken from the anterior half of the somite at the level of the developing forelimb (somites 13-14, Figure 4F). Consistent with the whole mount staining results, we identified Nrp1 within migrating NCCs, the dorsal aorta, and the periaortic mesenchyme. Nrp2 was also identified in NCCs and NCC precursors within the neural tube, the floor plate, somitic mesenchyme, and anterior cardinal veins. Notably, NCCs located around the dorsal aorta that had sympathetic fate preferentially expressed Nrp1 (Figure 4Fii, white arrow). Moreover, NCCs with presumptive sympathetic fate migrating towards the dorsal aorta also preferentially expressed high levels of Nrp1 (Figure 4Fii and inset). By contrast, NCCs migrating within the somite and/or arresting within the anlagen of the DRG expressed various combinations of the neuropilin receptors. Although the majority of the NCCs within the area of the DRG co-expressed Nrp1 and Nrp2, we also identified NCCs within and around the DRG that preferentially expressed either Nrp1 (Figure 4Fi, arrow) or Nrp2 alone (Figure 4Fi and inset). NCCs expressing only Nrp1 were mostly located at the ventral side of the DRG in close association with the neural tube, while
NCCs expressing only Nrp2 were located at the prospective dorsal root entry zone.

We tested the notion that NCCs express combinations of the neuropilin receptors by purifying GFP-positive trunk NCCs from E9.5 Wnt1Cre; Z/EG embryos, using fluorescence-activated cell sorting (FACS). Consistent with our in vivo expression analysis, counterstaining of Nrp1 and Nrp2 identified three populations of trunk NCCs based on their neuropilin expression profiles: 1) Nrp1 high/Nrp2 high, 2) Nrp1 high/Nrp2 low, and 3) Nrp2 high/Nrp1 low (Figure 5). Given the different locations of these cells within the embryo, our expression profiling also suggests that each of these NCC types may give rise to different derivatives. We therefore propose that the majority of Nrp1 high/Nrp2 high NCCs form neurons and glia of the DRG, Nrp2 high/Nrp1 low NCCs form boundary cap cells at the dorsal root entry zone, and Nrp1 high/ Nrp2 low NCCs form neurons and glia of the sympathetic nervous system.

Upon reaching the dorsal aorta, NCCs commence differentiation into bona fide sympathetic neurons via the elevated expression of the basic helix-loop-helix transcription factor MASH1. NCC derivatives that have initiated this differentiation program also begin to express Nrp2 [37]. Consistent with this developmental progression, our expression analysis in sections from more developmentally advanced regions of E9.5 embryos (somites 5-6 that are anterior to the forelimb) identified a small number 

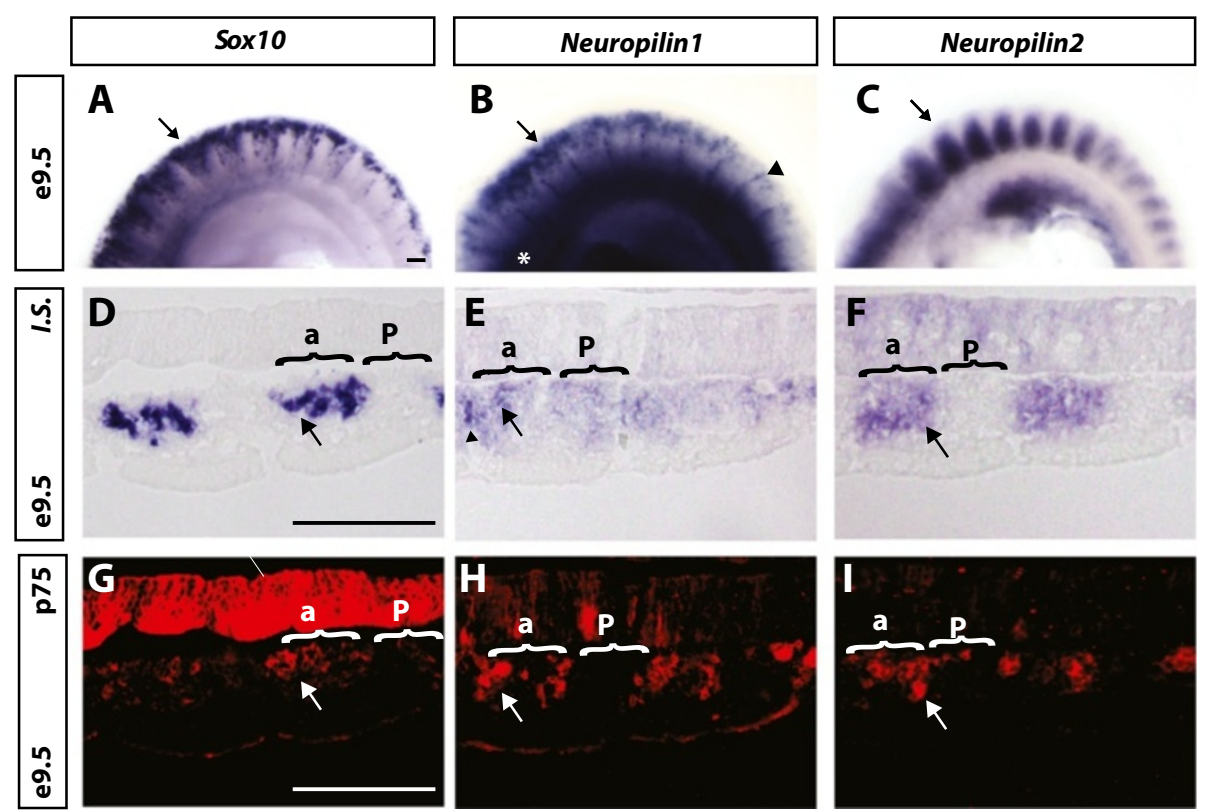

Figure 3 Nrp1 and Nrp2 are expressed in trunk NCCs at the same axial level. (A-C) Lateral view of whole mount in situ hybridisation at E9.5 for Sox 10, Nrp1, and Nrp2. (A) Sox10 whole mount in situ hybridisation specifically labelled NCCs migrating through the anterior half of the somite (black arrow). (B) Nrp1 in situ hybridisation labelled NCCs (black arrow), intersomitic blood vessels (white arrowhead) and the dorsal aorta (asterisks). (C) Nrp2 was expressed within the anterior half of the somite (black arrow). (D-I) Longitudinal sections of Nrp1 and Nrp2 in situ hybridisation over the hind limb counterstained with antibodies to p75. (D, G) p75 expression labelled Sox10-positive NCCs in the anterior half of the somite (white and black arrow, respectively). (E, H) Nrp1 had its highest expression in cells that also expressed p75 (black and white arrows), and was also expressed in the somitic tissue and intersomitic blood vessels. (F, I) Nrp2 was expressed in the anterior half of the somite within NCCs and also in the somitic tissue (black and white arrows). a, anterior; IS, in situ hybridisation; p, posterior. Scale bars $=100 \mu \mathrm{m}$.

of GFP-positive cells at the dorsal aorta co-expressing Nrp1 and Nrp2 (not shown).

Previous phenotypic analysis of neuropilin knockout embryos found that Nrp1 is required for correct positioning of both sensory and sympathetic neurons, while Nrp2 is required for positioning of sensory neurons [28,33,34]. Our expression analysis therefore explains the disparate phenotypes between the two types of knockout mice, and further suggests that neuropilins may play essential roles in coordinating NCC migration with fate specification.

\section{Generation of an inducible lineage-tracing Nrp2 transgenic mouse model}

To identify the fate of neuropilin-expressing NCCs and to determine if Nrp2-expressing trunk NCCs are biased towards the sensory neuronal lineage, we generated a tamoxifen-inducible Nrp2 lineage-tracing mouse model. A Nrp2-CreERT2/Kikume transgenic construct was generated by replacing the ATG start codon of the Nrp2 gene with the entire CreERT2 IRES Kikume sequence followed by a polyadenylation signal sequence, thus placing CreERT2 IRES Kikume under control of the endogenous regulatory elements contained in the Nrp2 gene locus (Figure 6A). The engineered construct was used to generate a transgenic founder mouse that was identified by PCR using primers specific for CreERT2.
The offspring from the founder line appeared grossly normal, and the transgene was transmitted as predicted by Mendelian ratios.

To permanently label and follow the fate of $N r p 2$ expressing cells throughout embryogenesis, Nrp2-CreERT2/ Kikume transgenic mice were crossed with $R 26 R$ LacZ reporter mice to create Nrp2-CreERT2/Kikume; R26R LacZ embryos. Upon addition of tamoxifen to these embryos, CreERT2 translocated to the nucleus to promote stable expression of $\beta$-galactosidase (lacZ) in cells and descendants of cells endogenously expressing $N r p 2$ at the time of injection. No lacZ labelling was detected without tamoxifen injection (data not shown).

We next determined that intraperitoneal injection of tamoxifen at $2-4 \mathrm{mg}$ per $40 \mathrm{~g}$ body weight to pregnant dams was sufficient to label Nrp2-expressing cells without resulting in embryonic lethality. As expected, higher doses of tamoxifen injected at E7.5 to E8.5 had detrimental effects on embryos.

Having optimised the dosage of tamoxifen, we first validated that the transgene recapitulated the Nrp2 expression profile in NCCs. Tamoxifen was injected at E9.0 and E9.5, and embryos collected at E10.0. Analysis of Xgal staining in whole mount embryos demonstrated that the transgene is expressed in a highly overlapping pattern to endogenous $\operatorname{Nrp} 2(n=12$; compare Figure $6 \mathrm{~B}$ 

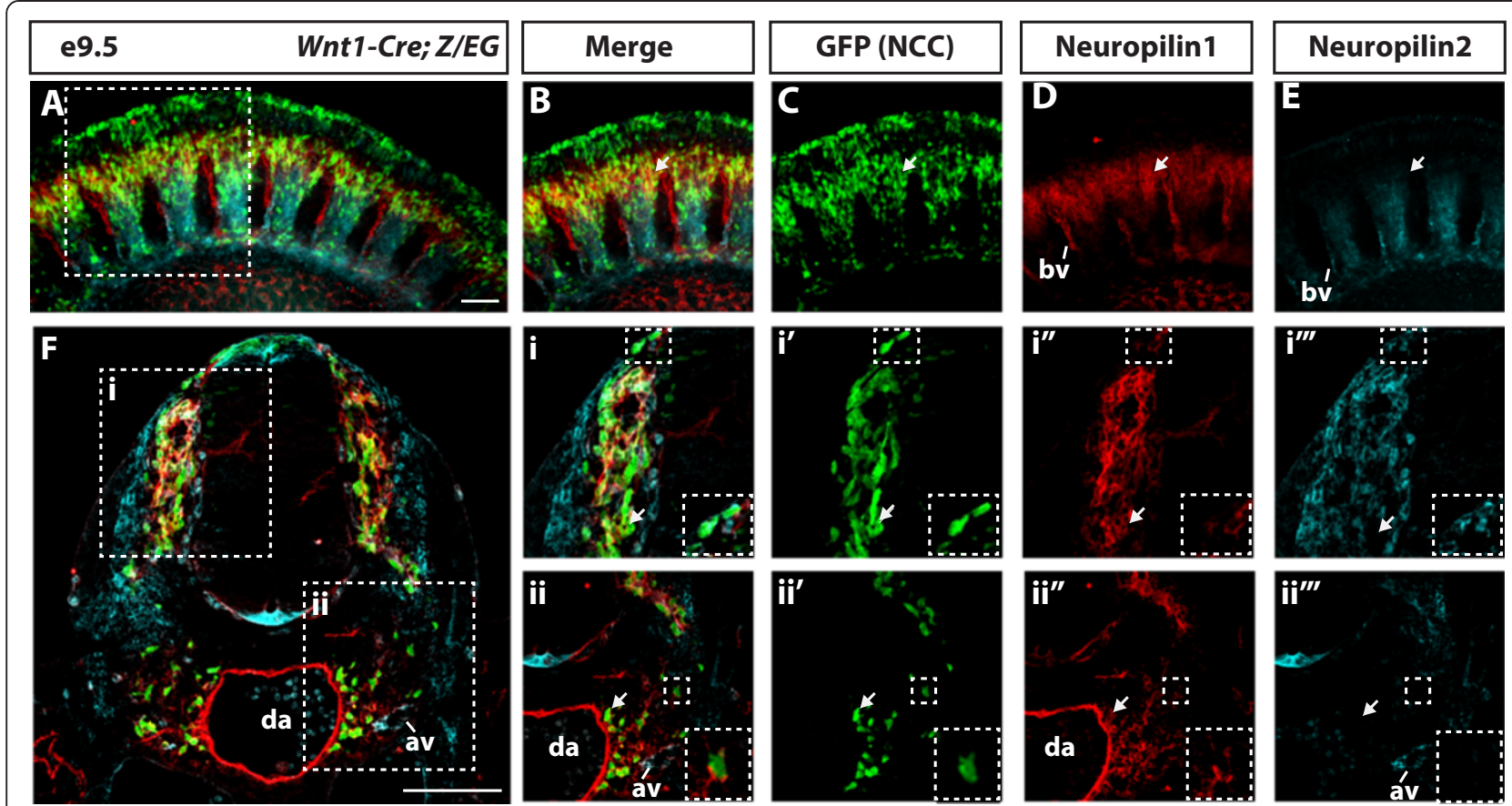

Figure 4 Nrp1 and Nrp2 are expressed in distinct populations of trunk NCCs. (A-E) Lateral view of whole mount immunofluorescence staining of Nrp1, Nrp2 and trunk NCCs at E9.5. (C) GFP labelled all NCCs migrating in the anterior half of the somite. (D) Nrp1 was expressed in many GFP-positive NCCs and intersomitic vessels. (E) Nrp2 was expressed in the anterior half of the somite and within NCCs. Notably, Nrp1 had high expression in NCCs that had either low or negative Nrp2 expression (white arrow). (F) Transverse sections through the anterior half of the somite over the forelimb region showed that neuropilins were expressed within different populations of NCCs. NCCs migrating through the somite in and around the anlage of the dorsal root ganglia (Fi-i'"') consisted of cells with high Nrp2 and no Nrp1 expression (inset) and conversely, with high levels of Nrp1 and no expression of Nrp2 (white arrow). (Fii-ii"') NCCs migrating towards the dorsal aorta (inset) or condensing in the region of the sympathetic ganglia (white arrow) preferentially expressed Nrp1. av, anterior cardinal vein; bv, blood vessel; da, dorsal aorta. Scale bars $=100 \mu \mathrm{m}$.

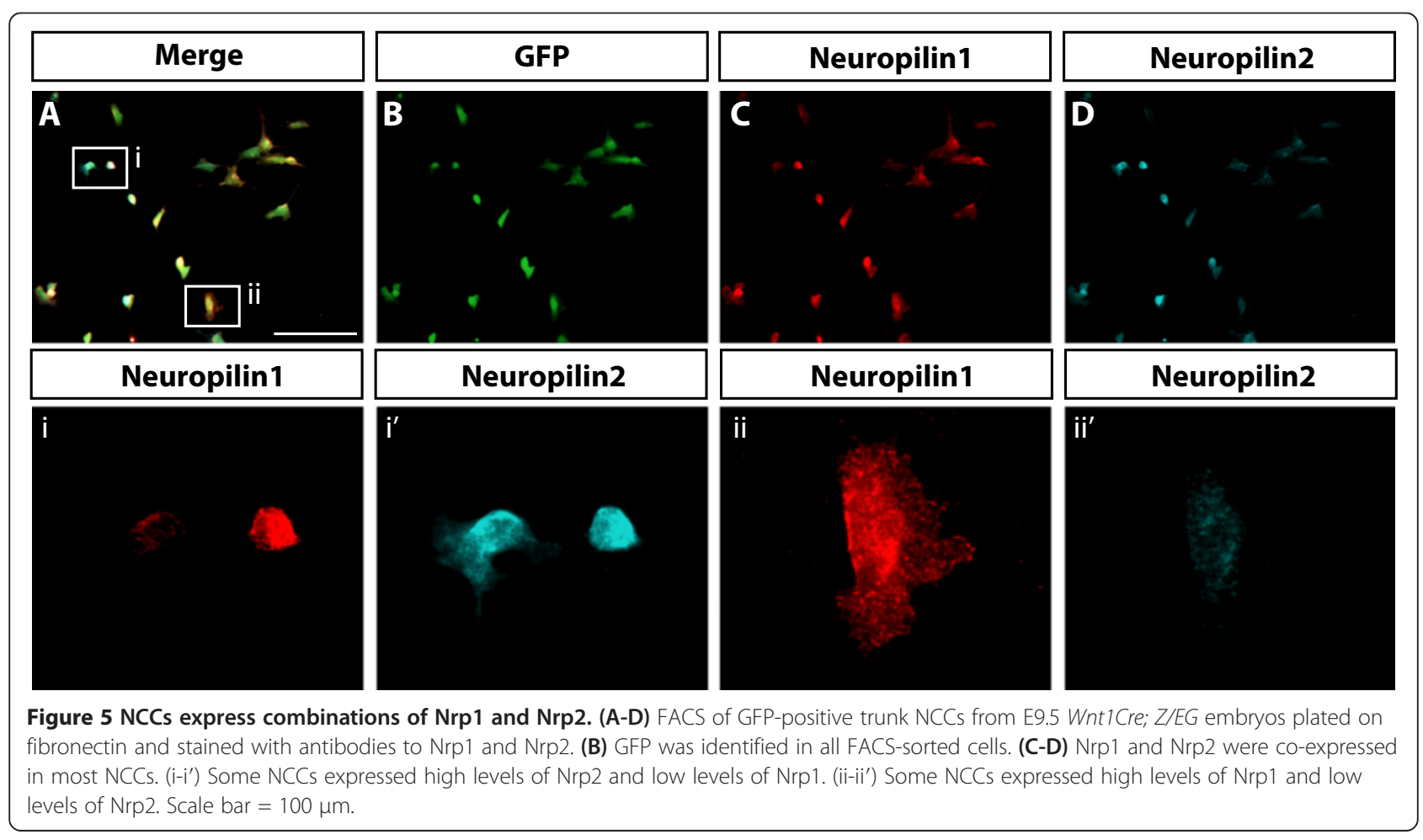




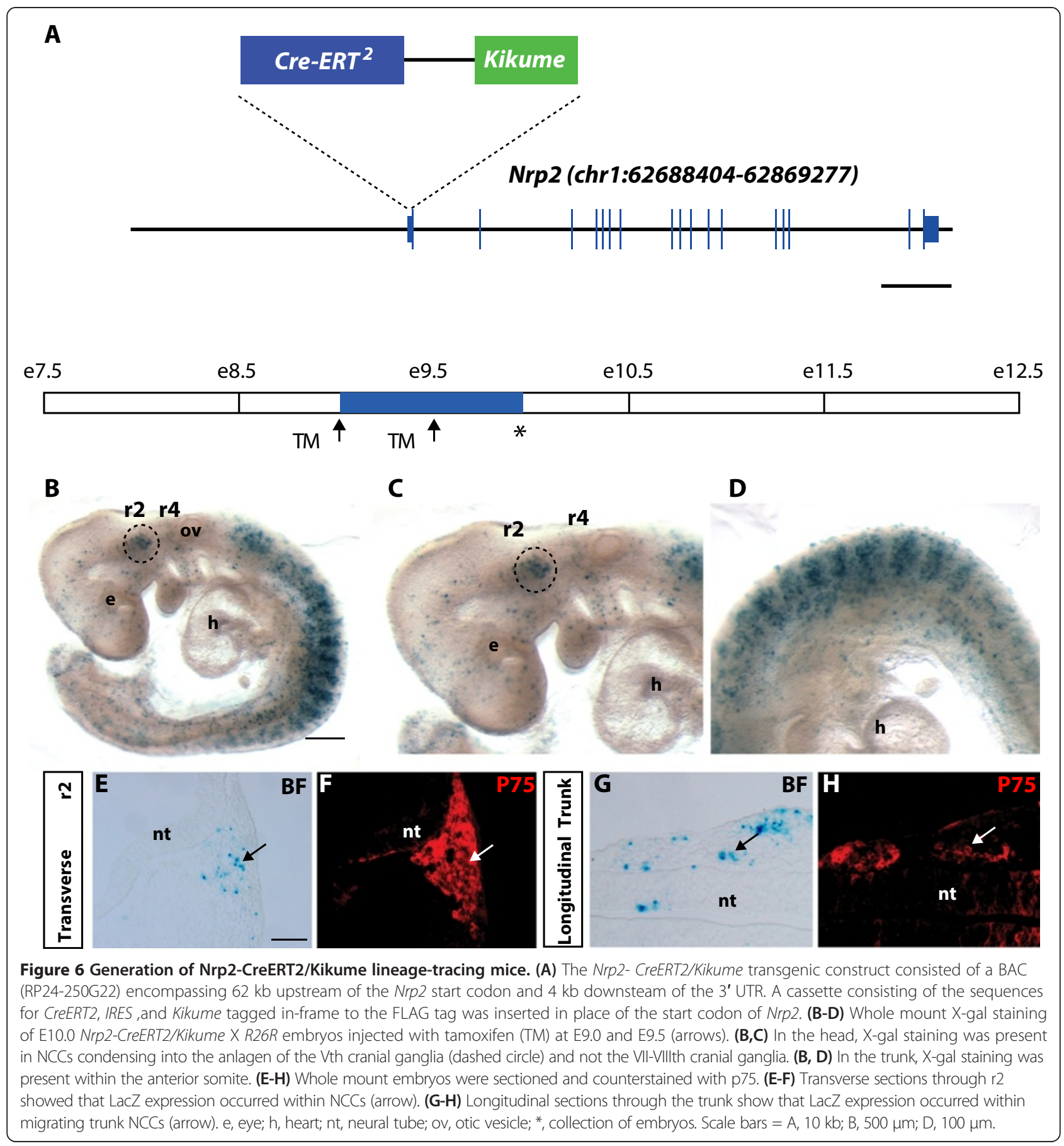

with Figures 1I, 2D, 3C, and 4E). Indeed, the transgene expression pattern replicated the expression of Nrp2 within a distinct striped pattern in the trunk and within NCCs migrating out of r2 (Figure 6B-D). To confirm that the expression was occurring within NCCs, we sectioned E10.0 embryos and counterstained with the NCC marker p75 (Figure 6E-H). Transverse sections through $\mathrm{r} 2$ and longitudinal sections through the trunk confirmed transgene expression within migrating NCCs, somitic tissue, and other cell types within the epidermis (detailed analysis of transgene expression in other tissues will be presented elsewhere). These findings demonstrate that the regulatory elements controlling expression of Nrp2 within NCCs are present within the bacterial artificial chromosome (BAC) used to make this transgenic animal. This mouse model provides an ideal resource for lineage-tracing studies and for the temporal removal of floxed genes in specific NCC 
populations and other cell types in which Nrp2 is expressed.

\section{Nrp2-expressing NCCs give rise to neurons and glia in the trigeminal ganglia}

Using this inducible lineage-tracing mouse model, we first determined the derivatives of Nrp2-expressing NCCs in the head. Tamoxifen was administered to pregnant dams when embryos were at E9.5 and E10.0, and later collected at E11.5. Consistent with Nrp2 being restricted to specific cranial NCC subpopulations, analysis of whole mount embryos revealed distinct X-gal staining in the trigeminal $(\mathrm{V})$ ganglia that are derived from NCCs migrating out of $\mathrm{r} 2(\mathrm{n}=8$, Figure $7 \mathrm{~A})$. By contrast, minimal staining was detected in $\mathrm{r} 4 \mathrm{NCC}$ derived structures such as the facio-acoustic ganglia (VII-VIII). X-gal staining also identified additional Nrp2-expressing cells in the forebrain region. Longitudinal sections counterstained with eosin confirmed the restriction of Nrp2-expressing NCCs to the trigeminal ganglia (V) (Figure 7B-C).

To define the fate of Nrp2-expressing NCCs, we next counterstained longitudinal X-gal-stained sections with antibodies specific for neurons (anti-Tuj1 antibodies) and glia (anti-Sox9 antibodies) (Figure 7D-G). Under high magnification, Nrp2-expressing NCCs were found to give rise to both Tuj1-positive neurons and Sox9-positive glia within the trigeminal ganglia (V) (Figure 7Gi, Gii, respectively). Taken together with our expression profiling results, we conclude that Nrp2 expression is restricted to NCCs emigrating out of hindbrain $\mathrm{r} 2$. As we were unable to detect X-gal staining in other derivatives of the r2 stream such as bone and cartilage of the mandible, our results suggest that Nrp2-expressing NCCs preferentially give rise to neuroglial lineages. In addition, both whole mount staining and staining of transverse sections (not shown), suggest that Nrp2-expressing NCCs give rise to

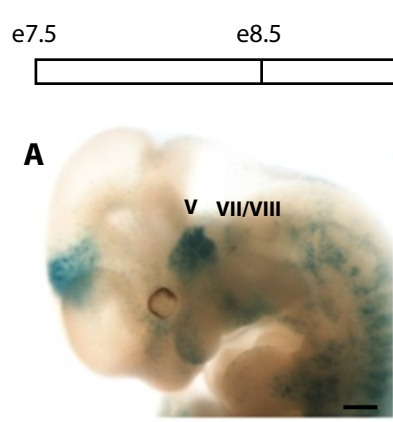

\begin{tabular}{cccc} 
e9.5 & e10.5 & e11.5 & e12.5 \\
\hline & & & \\
\hline
\end{tabular}
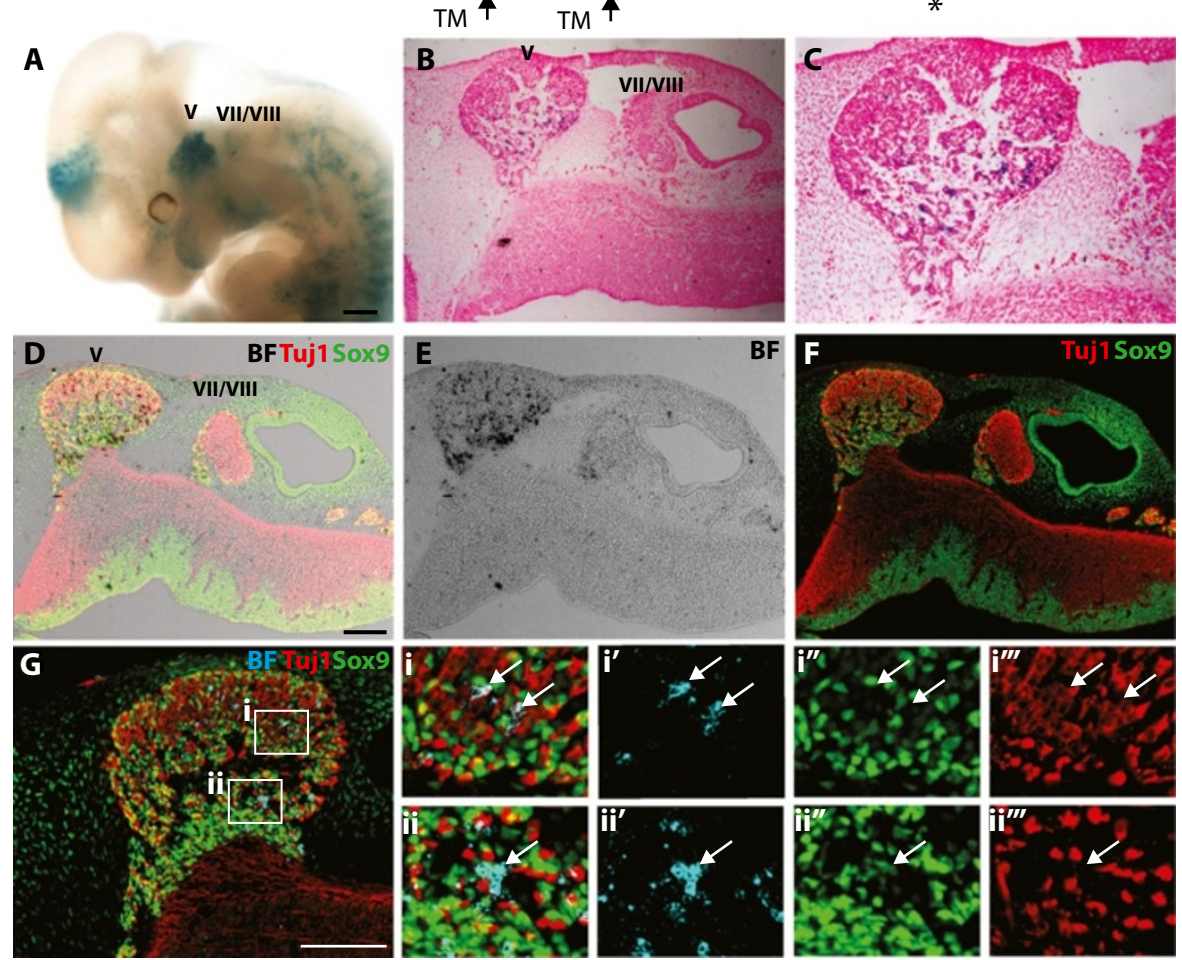

Figure 7 Nrp2-expressing NCCs give rise to neurons and glia of the trigeminal ganglia. (A) Whole mount X-gal staining of E11.5 Nrp2CreERT2/Kikume X R26R embryos injected with tamoxifen (TM) at E9.5 and E10.0 (arrows). Staining was restricted to the trigeminal ganglia (V) that were derived from the $\mathrm{r} 2$ stream of NCCs. Notably, staining was absent from derivatives of the $\mathrm{r} 4$ stream of NCCs such as the facio-acoustic ganglia (VII-VIII). (B-C) Longitudinal sections through the head of Nrp2-CreERT2/Kikume X R26R embryos counterstained for X-gal and eosin confirmed that transgene expression was restricted to the trigeminal ganglia ( $V$ ) and lacking in the facio-acoustic ganglia (VII-VIII). (D-G) Serial sections to that in (B) counterstained for $\mathrm{X}$-gal, Tuj1, and Sox9 confirmed transgene expression within neurons and glia of the trigeminal ganglia ( $V$ ) and not within the facio-acoustic ganglia (VII-VIII). (D) Bright-field (BF) images of X-gal staining overlaid with Tuj1 and Sox9. (G) BF images were colour-inverted to cyan to promote colocalisation analysis. (Gi-Gi'”' X-gal-positive descendants of Nrp2-expressing NCCs co-expressed Tuj1 (arrow). (Gii-Gii'”') X-gal-positive descendants of Nrp2-expressing NCCs co-expressed Sox9 (arrow). Scale bars = A, $500 \mu \mathrm{m} ; \mathrm{D}, \mathrm{G}, 100 \mu \mathrm{m}$. 
Schwann cells along the axonal tracts of the trigeminal ganglia (V).

\section{Nrp2-expressing trunk NCCs are biased towards sensory ganglia}

Our expression analysis raised the hypothesis that ventrally migrating trunk NCCs expressing Nrp2 are biased towards neurons and glia of the sensory ganglia. To test this notion, we completed fate-mapping studies with the Nrp2-inducible lineage-tracing mouse model. Tamoxifen was administered to pregnant dams when embryos were E9.5 and E10.0, and these were later collected at E11.5, a stage at which the sensory and sympathetic ganglia have started to condense. Consistent with the staining of Nrp2 in NCCs and paraxial mesoderm at E9.5, X-gal staining identified derivatives of Nrp2-expressing cells within the DRG and skeletal muscle (Figure 8A). Eosin staining of longitudinal sections anterior to the hind limb clearly demonstrated X-gal staining within the DRG (Figure 8B-C). Transverse sections through the same axial level were also counterstained with the pan neuronal marker Tuj1 and the sympathetic specific neuronal marker tyrosine hydroxylase (TH) (Figure 8D). In all cases examined $(n=3)$, X-gal staining was robustly observed in the DRG (Figure 8D-F). By contrast, we were unable to observe $\mathrm{X}$-gal staining in sympathetic ganglia (Figure 8G-I). This finding demonstrates that Nrp2-expressing NCCs are strongly biased towards neurons and glia of the DRG.

Our fate-mapping results of Nrp2-expressing NCCs are strikingly similar to that presented for lineage tracing of Ngn2-expressing NCCs in mice [17]. In the latter, Ngn2-positive cells were also biased towards sensory ganglia. As Ngn2 is a basic helix-loop-helix transcription factor that plays an essential role in sensory neuronal differentiation [38], it will be of interest to determine if $\mathrm{Nrp} 2$ is also involved in the same molecular pathway within NCCs.

\section{Conclusions}

The work presented here identified molecularly distinct populations of cranial and trunk NCCs based on their expression profiles of the cell surface receptors Nrp1 and Nrp2. In combination with the recent analyses of

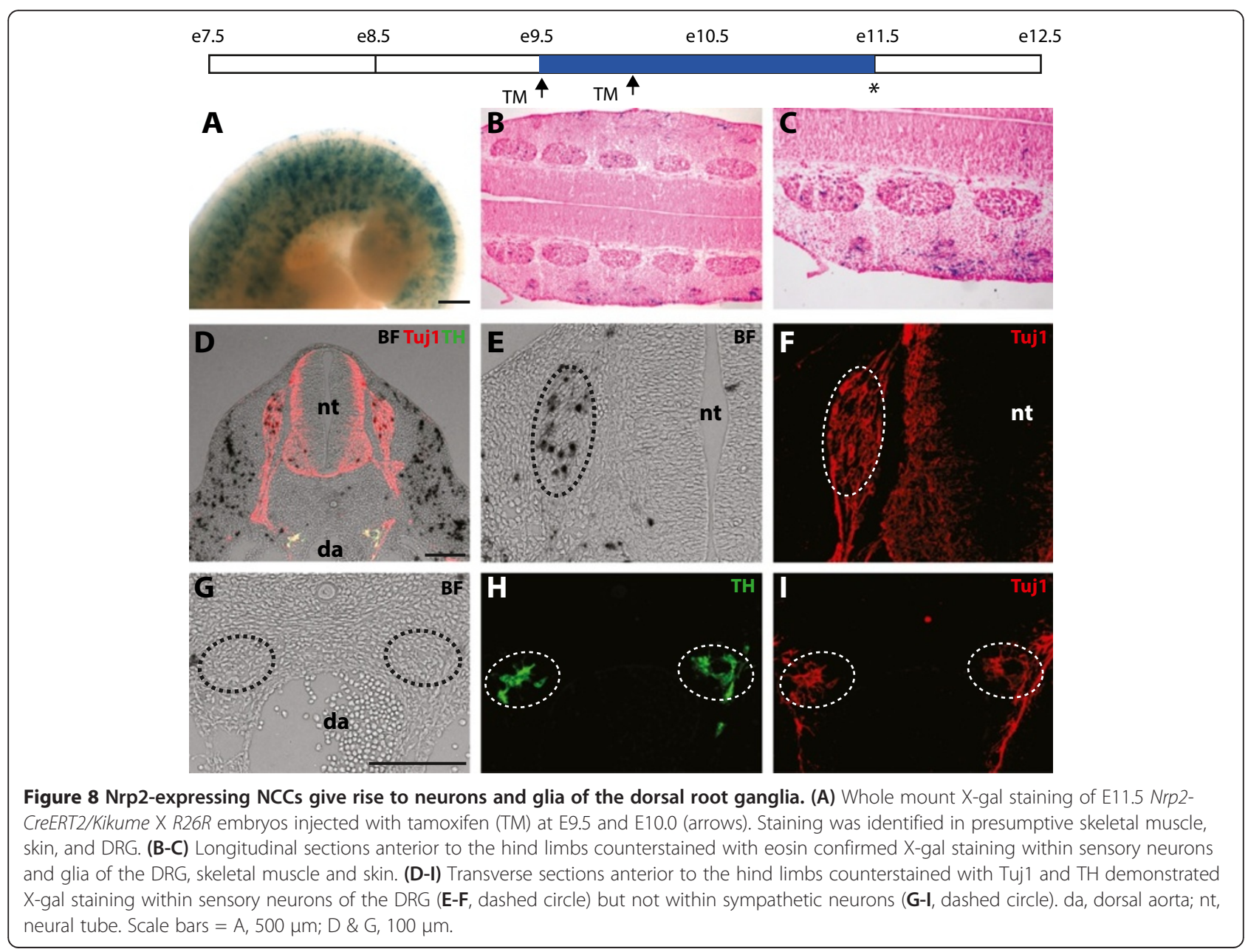


Nrp1 and Nrp2 knockout mice [31-34], this work further identifies essential roles for neuropilins in sorting specific populations of NCCs to their final destinations within the embryo (Figure 9A). In the head, Nrp1 is expressed in NCCs emigrating from $\mathrm{r} 4$, and is required for the migration and condensation of neurons and glia of the facio-acoustic ganglion (VII-VIII) [31]. By contrast, Nrp2 is expressed in NCCs emigrating from r2, and is required for the migration and condensation of neurons and glia of the trigeminal ganglion (V) [32].

In addition to their differential expression in restricted populations of NCCs along the anteroposterior axis, neuropilins also label divergent populations of NCCs along the dorsoventral axis (Figure 9A). Trunk NCCs migrating along the ventral path through the anterior somite consist of a mixed population of sympathetic and sensory progenitors. Our finding that Nrp1 and Nrp2 expression correlates with sympathetic and sensory progenitors, and that Nrp2-expressing NCCs are biased towards neurons and glia of the sensory nervous system therefore suggests that neuropilins, possibly in combination with other guidance molecules such as CXCR4 [25], provide part of the molecular machinery to guide ventrally migrating NCC precursors to correct target regions of the embryo (Figure 9B). Indeed, phenotypic analysis of neuropilin knockout mice is in strong agreement with this notion $[33,34]$.

Our study identified three distinct NCC sub-types within and around the DRG that can be segregated based on their neuropilin expression profiles. While Nrp2expressing NCCs (Nrp2-high/Nrp1-high and Nrp2-high/ Nrp1-low) were traced to the DRG it will be important to complete the inverse experiment with lineage tracing of Nrp1-expressing NCCs to determine if this population is also fate-restricted.

Our expression analysis also identified Nrp2 within presumptive NCC precursors in the dorsal neural tube (Figure 4F). Given that Nrp2 knockout embryos have defects restricted to sensory NCC derivatives, and that Nrp2-expressing NCCs are fate-restricted towards a
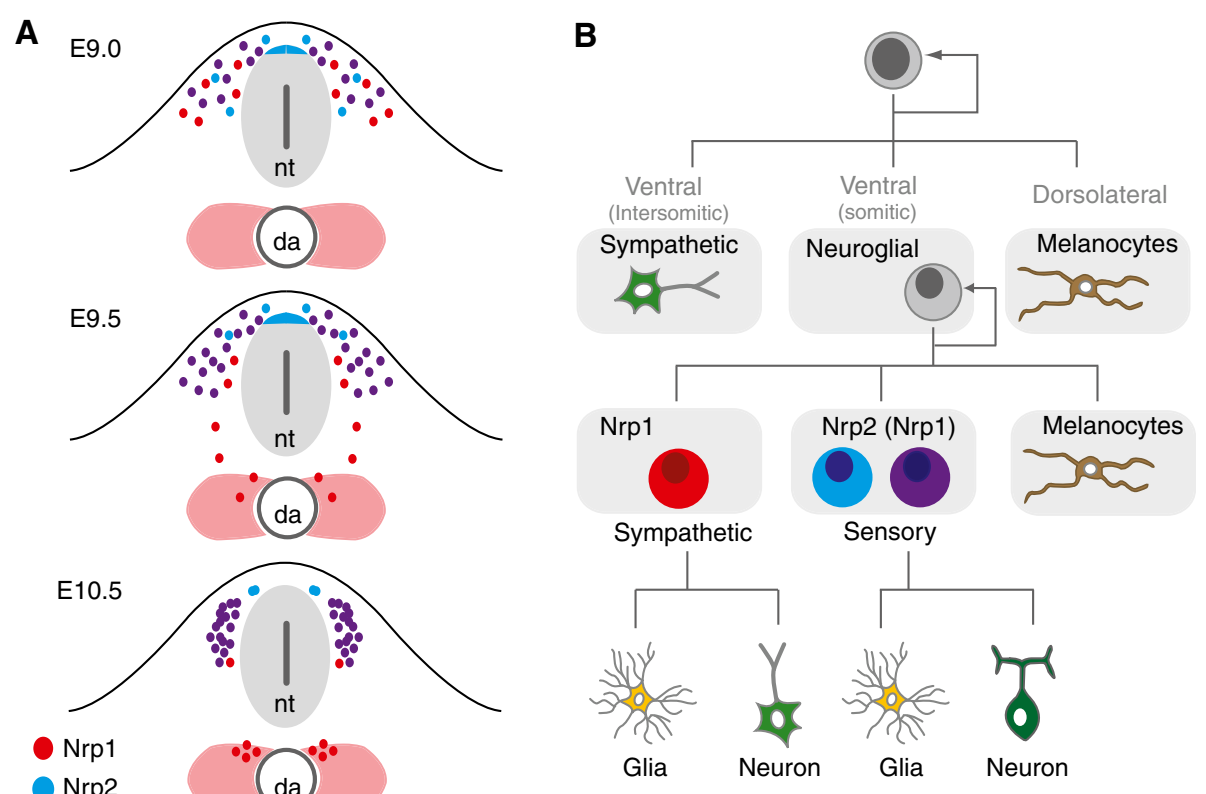

Figure 9 Neuropilins define molecularly distinct populations of ventrally migrating trunk NCCs. (A) Schematic detailing the neuropilin expression profiles in migrating trunk NCCs. NCCs migrating to the dorsal aorta (da) give rise to the sympathetic ganglia, while NCCs that stall within the somite alongside the neural tube (nt) give rise to sensory neurons of the DRG. Trunk NCCs at the level of the forelimb begin to delaminate from the neural tube at E8.5, and by E9.0, have started to migrate within the somite. At E9.5, some NCCs have already reached the dorsal aorta, while others have stalled in the anlagen of the DRG. At E10.5, NCCs with sympathetic fate have condensed, while NCCs with sensory fate have started differentiating in the DRG. Expression profiling identified distinct populations of NCCs with Nrp1 (red), Nrp2 (blue), and Nrp1/Nrp2 (purple). Nrp1-expressing cells preferentially migrate towards the dorsal aorta while Nrp2-expressing cells stall within the area of the DRG. Nrp2 was also expressed in presumptive NCC precursors within the dorsal neural tube. (B) Schematic diagram detailing the fate restriction of trunk NCCS. NCCs delaminate from the neural tube to migrate along two separate paths. Ventrally migrating NCCs initially travel through the intersomitic space to seed the sympathetic ganglia, and then switch to travel ventrally through the anterior half of the somite to give rise to sensory ganglia, sympathetic ganglia, and melanocytes. NCCs migrating dorsolaterally also give rise to melanocytes. Expression, fate-mapping, and phenotypic studies suggest that Nrp2 (Nrp2 alone (blue) and Nrp1/Nrp2 (purple)) is a marker of NCCs biased towards sensory ganglia. In addition, expression and phenotypic studies suggest that Nrp1 (red) is a marker of ventrally migrating NCCs that give rise to sympathetic ganglia. 
sensory phenotype, our data could also be taken to support the notion that at least some pre-migratory NCCs are fate-biased prior to delamination. However, as clonal analysis or transplantation of Nrp2-expressing premigratory/migrating NCCs into ectopic environments has not yet been achieved, our studies are currently unable to decipher between the two models of when ventrally migrating NCCs are segregated into specified lineages. For example, although the Nrp2-expressing cells may be biased towards a sensory phenotype, they may only commit to this lineage upon reaching their final resting place within the sclerotome.

Taken together with previous reports, our studies demonstrate that neuropilins provide part of the molecular machinery to sort heterogeneous populations of ventrally migrating NCCs towards correct target regions.

\section{Methods}

\section{Mice}

All experimentation was approved by and conducted in accordance with the guidelines of the Animal Ethics Committee of the SA Pathology/Central Adelaide Local Health Network, and followed the Australian code of practice for the care and use of animals for scientific purposes. To obtain embryos of defined gestational ages, animals were mated in the evening, and the morning of vaginal plug formation was counted as embryonic day (E) 0.5. To lineage-trace NCCs and their derivatives throughout development, we crossed Wnt1Cre [35] with Z/EG [36] mice.

\section{Generation of Nrp2-CreERT2/Kikume transgenic mice}

The BAC clone RP24-250G22, containing $62 \mathrm{~kb}$ of DNA upstream of the first exon to $4 \mathrm{~kb}$ past the final coding exon of the Nrp2 locus, was obtained from the BAC resource at Children's Hospital Oakland Research Institute (Oakland, California, USA). A CreERT2 IRES FLAGKikume cassette was inserted at the initiation codon of the Nrp2 coding sequence, which is located within exon 1 , by homologous recombination using a targeting vector designed for BAC recombineering [39,40]. A 5' homology arm was amplified by PCR with a forward primer $5^{\prime}$-taactagtctcgagctctgggaacacagagctgag- $3^{\prime}$ and reverse primer $5^{\prime}$-taactagtagagagcgatccgattacg- 3 ' and inserted upstream of the CreERT2 IRES FLAG-Kikume sequence in plasmid pBS-CreERT2 IRES FLAG-Kikume (Schwarz laboratory). The 5' homology arm and CreERT2 IRES FLAG-Kikume fragment was then sub-cloned to the recombineering vector PL451 using XhoI restriction sites. A 3' homology arm was amplified by PCR with a forward primer $5^{\prime}$-taggatccgtaagcccttcaaagtttttc-3' and reverse primer 5 '-tagcggccgcaaagaatccacacatgtgaaaag-3', and inserted downstream of CreERT2 IRES FLAG-Kikume $\mathrm{Neo} / \mathrm{Kan}$ in the PL451 vector using unique BamHI/NotI sites. This created the Nrp2- CreERT2/Kikume PL451 recombineering vector.

The RP24-250G22 BAC was transformed into the recombinogenic Escherichia coli strain EL250 and maintained with chloramphenicol resistance. EL250 cells carrying RP24-250G22 BAC were electroporated with Nrp2-CreERT2/Kikume PL451, and homologous recombined positive clones were selected with kanamycin and chloramphenicol. The Neo/Kan cassette was removed by $\mathrm{L}$-arabinose induction of flp recombinase, resulting in a Nrp2-CreERT2/Kikume BAC. Homologous recombination was confirmed by PCR and sequencing of the BAC using primers that flanked upstream of the $\mathrm{Nrp2} \mathrm{5}^{\prime}$ homology arm and downstream of the 3' homology arm. High-quality BAC DNA was prepared using the Large Construct Kit (Qiagen, Chadstone, VIC, Australia) and analysed by pulsed field gel electrophoresis, restriction analysis, and BAC end sequencing. Prior to microinjection, the DNA elution buffer was replaced with fresh microinjection buffer $(10 \mathrm{mM}$ Tris- $\mathrm{HCl}, \mathrm{pH} 8.0$ $0.25 \mathrm{mM}$ EDTA, pH 8.0) by microdialysis. The Nrp2CreERT2/Kikume BAC was microinjected into fertilized embryos by standard pronuclear injection techniques at the Transgenic Animal Services Queensland (TASQ, Brisbane, GLD, Australia). Genomic DNA was isolated from tail samples [41], and founder mice carrying the BAC transgene were identified by PCR with primer pairs recognising CreERT2: iCre forward $5^{\prime}$-gagagatggatctctgtgtc- 3 ' and iCre reverse $5^{\prime}$-gacttcatcagaggtggcatc-3', yielding a $580 \mathrm{bp}$ product. Founders and offspring were subsequently genotyped with the same primer pair. One founder line was produced that transmitted the transgene in a normal mendelian inheritance pattern, with all offspring appearing grossly normal.

\section{In situ hybridisation}

Whole mount and section in situ hybridisation was performed as described previously [42]. Riboprobes were transcribed from plasmids containing the cDNA sequence for Sox10, Nrp1, and Nrp2 [33,34].

\section{Immunolabelling}

Embryos were fixed in 4\% paraformaldehyde in PBS. All sections were cut at a thickness of $14 \mu \mathrm{m}$ on a CM1850 cryostat (Leica, North Ryde, NSW, Australia) and airdried for $60 \mathrm{~min}$ before staining. For immunolabelling, cryosections or whole mount embryos were blocked in PBS containing $0.2 \%$ BSA and $0.5 \%$ Triton X-100, and stained with the indicated primary antibodies: rabbit anti-Sox9 1:1000; rabbit tyrosine hydoxylase 1:300 (both Millipore, Batswater, VIC, Australia); goat anti-Nrp1 1:500; rabbit anti-Nrp2 1:500 (both R\&D, Minneapolis, MN, USA); rabbit anti-p75-NTR 1:200 (Epitomics, Burlingame, CA, USA); mouse anti $\beta$-tubulin isotype 
III (Sigma-Aldrich, Sydney, NSW, Australia) 1:750; and chicken anti-GFP 1:1000 (Abcam, Melbourne, VIC, Australia). Cyrosections were mounted in Prolong Gold Antifade Reagent containing DAPI (Molecular Probes, Mulgrave, VIC, Australia). Confocal images were acquired under a confocal microscope (LSM 700; Zeiss, Jena, Germany). All images were prepared with Photoshop software (Adobe, San Jose, CA, USA).

\section{$\beta$-Galactosidase staining}

Embryos were fixed in PBS containing 4\% paraformaldehyde. Whole embryos or cryosections were incubated in staining solution: $19 \mathrm{mM}$ sodium dihydrogen phosphate, $81 \mathrm{mM}$ disodium hydrogen phosphate, $2 \mathrm{mM} \mathrm{MgCl}_{2}$, 5 mM EGTA, $0.01 \%$ sodium deoxycholate, $0.02 \%$ NP-40, $5 \mathrm{mM}$ potassium ferricyanide, $5 \mathrm{mM}$ potassium ferrocyanide, and $1 \mathrm{mg} / \mathrm{ml} \mathrm{X-gal} \mathrm{substrate,} \mathrm{at} 37^{\circ} \mathrm{C}$ until blue staining was sufficient. Sections were counterstained with eosin.

\section{Tamoxifen}

Tamoxifen 1 g (Sigma-Aldrich, Sydney, NSW, Australia) was suspended in $5 \mathrm{ml}$ of ethanol, and then dissolved in $45 \mathrm{ml}$ of sunflower oil to produce a final stock at $20 \mathrm{mg} / \mathrm{ml}$ concentration. To dissolve the tamoxifen completely, the stock was sonicated in an ice bath for 5 min (with 30 second intervals). Pregnant dams were injected intraperitoneally with $2 \mathrm{mg}$ tamoxifen per $40 \mathrm{~g}$ body weight at the indicated time points.

\section{FACS sorting of primary neural crest cells}

Primary NCCs were isolated from E9.5 Wnt1Cre; Z/EG embryos as previously described [43]. Trunk regions posterior to somite 10 were dissociated using Tryple Express (Invitrogen, Mulgrave, VIC, Australia) for $10 \mathrm{mi}-$ nutes at room temperature. Dissociated cells were washed twice with Dulbecco's modified Eagle's media (DMEM) containing $10 \%$ fetal calf serum (FCS), and resuspended in DMEM with 1\% FCS for cell sorting. Cell sorting was performed on a Beckman Coulter Epics Altra HyperSort using Expo MultiComp Software (v1.2B; Beckman Coulter, Lane Cove, NSW, Australia) equipped with an Innova $300 \mathrm{C}$ water-cooled $488 \mathrm{~nm}$ argon laser at $100 \mathrm{~mW}$. Sorting was conducted at room temperature, with the instrument pressurised to $12 \mathrm{psi}$ and equipped with a $100 \mu \mathrm{m}$ nozzle. Linear forward scatter (FSC) peak signal (pk), width (time of flight (TOF)) and internal signal area (INT) signals were collected to allow for standard scatter and doublet discrimination. Linear side scatter (SSC) and INT signal were collected with a $488 / 10$ band pass filter in the photomultiplier tube (PMT1). Log GFP signal was collected in PMT2 with a 525/25 band pass filter behind a $488 \mathrm{~nm}$ long pass dichroic mirror. A gate was drawn on FSC versus SSC plot to exclude debris and dead cells, as discriminated by scatter properties alone. Following this, an FSC pk versus FSC INT plot was examined to allow distinction of single cells. Linearly related cells were gated for further analysis on a GFP versus SSC plot. Cells were collected into DMEM with 10\% FCS. After collection, primary NCCs were centrifuged, resuspended in NCC growth media (DMEM F12, 5\% chicken embryo extract, $10 \mathrm{mM}$ HEPES, 2\% B27, 1\% N2, $20 \mathrm{ng} / \mathrm{ml}$ insulin-like growth factor (IGF, R\&D, Minneapolis, MN, USA), and $100 \mathrm{U} / \mathrm{ml}$ Penicillin/Streptomycin (Invitrogen, Mulgrave, VIC, Australia). Cells were plated in this media in Ibidi $\mu$-slide eight-well dishes coated with $50 \mathrm{mg} / \mathrm{ml}$ fibronectin (Roche, Basel, Switzerland) at a concentration of $5 \times 10^{4}$ cells/well. After 4 hours, cells were fixed with 4\% PFA for 10 minutes, and immunolabelled with antiNrp1 and anti-Nrp2 antibodies. Images were acquired on an Olympus IX81 microscope using Cell X-cellence software (Olympus, Edwardstown, SA, Australia).

\section{Abbreviations}

BAC: bacterial artificial chromosome; DRG: Dorsal root ganglia; GFP: Green fluorescent protein; NCC: Neural crest cell; Nrp1: Neuropilin 1;

Nrp2: Neuropilin 2; TH: Tyrosine hydroxylase.

\section{Competing interests}

The authors declare that they have no competing interests.

\section{Authors' contributions}

$\mathrm{RL}, \mathrm{MS}, \mathrm{NH}$, and QS participated in the design, RL, MS, SW, SK, and QS executed and/or analysed all of the experiments, and RL and QS wrote the manuscript. All authors read and approved the final manuscript.

\section{Acknowledgements}

We thank X. Xu for help with genotyping mice and G. Secker for critical feedback. This work was supported by NHMRC project grant APP1008426.

\section{Author details}

${ }^{1}$ Centre for Cancer Biology, University of South Australia and SA Pathology, Frome Road, Adelaide 5000, Australia. ${ }^{2}$ Medical School, University of Adelaide, Frome Road, Adelaide 5000, Australia.

Received: 8 August 2014 Accepted: 14 October 2014 Published: 3 November 2014

\section{References}

1. Cordero DR, Brugmann S, Chu Y, Bajpai R, Jame M, Helms JA: Cranial neural crest cells on the move: their roles in craniofacial development. Am J Med Genet A 2010, 155A:270-279.

2. Bronner-Fraser M, Sieber-Blum M, Cohen AM: Clonal analysis of the avian neural crest: migration and maturation of mixed neural crest clones injected into host chicken embryos. J Comp Neurol 1980, 193:423-434.

3. KUO BR, Erickson CA: Vagal neural crest cell migratory behavior: a transition between the cranial and trunk crest. Dev Dyn 2011, 240:2084-2100.

4. Kirby ML, Gale TF, Stewart DE: Neural crest cells contribute to normal aorticopulmonary septation. Science 1983, 220:1059-1061.

5. Harris ML, Erickson CA: Lineage specification in neural crest cell pathfinding. Dev Dyn 2007, 236:1-19.

6. Ruhrberg C, Schwarz Q: In the beginning: generating neural crest cell diversity. Cell Adh Migr 2010, 4:622-630.

7. Krispin S, Nitzan E, Kalcheim C: The dorsal neural tube: a dynamic setting for cell fate decisions. Dev Neurobiol 2010, 70:796-812

8. Theveneau E, Mayor R: Neural crest migration: interplay between chemorepellents, chemoattractants, contact inhibition, epithelialmesenchymal transition, and collective cell migration. Wiley Interdiscip Rev Dev Biol 2012, 1:435-445. 
9. Trainor PA, Krumlauf R: Hox genes, neural crest cells and branchial arch patterning. Curr Opin Cell Biol 2001, 13:698-705.

10. Adameyko I, Lallemend F, Aquino JB, Pereira JA, Topilko P, Muller T, Fritz N, Beljajeva A, Mochii M, Liste I, Usoskin D, Suter U, Birchmeier C, Ernfors P: Schwann cell precursors from nerve innervation are a cellular origin of melanocytes in skin. Cell 2009, 139:366-379.

11. Harris ML, Hall R, Erickson CA: Directing pathfinding along the dorsolateral path - the role of ednrb2 and ephb2 in overcoming inhibition. Development 2008, 135:4113-4122.

12. Santagati F, Rijli FM: Cranial neural crest and the building of the vertebrate head. Nat Rev Neurosci 2003, 4:806-818.

13. Thomas AJ, Erickson CA: Foxd3 regulates the lineage switch between neural crest-derived glial cells and pigment cells by repressing mitf through a non-canonical mechanism. Development 2009, 136:1849-1858,

14. Jia $L$, Cheng $L$, Raper J: Slit/Robo signaling is necessary to confine early neural crest cells to the ventral migratory pathway in the trunk. Dev Biol 2005, 282:411-421.

15. Reedy MV, Johnson RL, Erickson CA: The expression patterns of C-Kit and $\mathrm{SI}$ in chicken embryos suggest unexpected roles for these genes in somite and limb development. Gene Expr Patterns 2003, 3:53-58.

16. Wehrle-Haller B, Weston JA: Soluble and cell-bound forms of steel factor activity play distinct roles in melanocyte precursor dispersal and survival on the lateral neural crest migration pathway. Development 1995, 121:731-742.

17. Zirlinger M, Lo L, Mcmahon J, Mcmahon AP, Anderson DJ: Transient expression of the Bhlh factor neurogenin-2 marks a subpopulation of neural crest cells biased for a sensory but not a neuronal fate. Proc Natl Acad Sci U S A 2002, 99:8084-8089.

18. George L, Chaverra M, Todd V, Lansford R, Lefcort F: Nociceptive sensory neurons derive from contralaterally migrating, fate-restricted neural crest cells. Nat Neurosci 2007, 10:1287-1293.

19. Krispin S, Nitzan E, Kassem Y, Kalcheim C: Evidence for a dynamic spatiotemporal fate map and early fate restrictions of premigratory avian neural crest. Development 2010, 137:585-595.

20. Mckinney MC, Fukatsu K, Morrison J, Mclennan R, Bronner ME, Kulesa PM: Evidence for dynamic rearrangements but lack of fate or position restrictions in premigratory avian trunk neural crest. Development 2013, 140:820-830.

21. Bronner-Fraser M, Fraser S: Developmental potential of avian trunk neural crest cells in situ. Neuron 1989, 3:755-766.

22. Bronner-Fraser M, Fraser SE: Cell lineage analysis reveals multipotency of some avian neural crest cells. Nature 1988, 335:161-164.

23. Bronner-Fraser M, Fraser SE: Cell lineage analysis of the avian neural crest. Development 1991, (Suppl 2):17-22.

24. Saito D, Takase $Y$, Murai $H$, Takahashi $Y$ : The dorsal aorta initiates a molecular cascade that instructs sympatho-adrenal specification. Science 2012, 336:1578-1581.

25. Kasemeier-Kulesa JC, Mclennan R, Romine MH, Kulesa PM, Lefcort F: Cxcr4 controls ventral migration of sympathetic precursor cells. J Neurosci 2010, 30:13078-13088.

26. Schwarz Q, Ruhrberg C: Neuropilin, you gotta let me know: should I stay or should I go? Cell Adh Migr 2010, 4(1):61-66.

27. Roffers-Agarwal J, Gammill LS: Neuropilin receptors guide distinct phases of sensory and motor neuronal segmentation. Development 2009, 136:1879-1888.

28. Schwarz Q, Maden CH, Davidson K, Ruhrberg C: Neuropilin-mediated neural crest cell guidance is essential to organise sensory neurons into segmented dorsal root ganglia. Development 2009, 136:1785-1789.

29. Mclennan R, Kulesa PM: In vivo analysis reveals a critical role for neuropilin-1 in cranial neural crest cell migration in chick. Dev Biol 2007, 301:227-239.

30. Mclennan R, Teddy JM, Kasemeier-Kulesa JC, Romine MH, Kulesa PM: Vascular endothelial growth factor (Vegf) regulates cranial neural crest migration in vivo. Dev Biol 2010, 339(1):114-125.

31. Schwarz Q, Vieira JM, Howard B, Eickholt BJ, Ruhrberg C: Neuropilin 1 And 2 control cranial gangliogenesis and axon guidance through neural crest cells. Development 2008, 135:1605-1613.

32. Gammill LS, Gonzalez C, Bronner-Fraser M: Neuropilin 2/Semaphorin $3 f$ signaling is essential for cranial neural crest migration and trigeminal ganglion condensation. Dev Neurobiol 2007, 67:47-56.
33. Gammill LS, Gonzalez C, Gu C, Bronner-Fraser M: Guidance of trunk neural crest migration requires neuropilin 2/Semaphorin 3 f signaling. Development 2006, 133:99-106.

34. Schwarz Q, Maden CH, Vieira JM, Ruhrberg C: Neuropilin 1 signaling guides neural crest cells to coordinate pathway choice with cell specification. Proc Natl Acad Sci U S A 2009, 106:6164-6169.

35. Jiang X, Choudhary B, Merki E, Chien KR, Maxson RE, Sucov HM: Normal fate and altered function of the cardiac neural crest cell lineage in retinoic acid receptor mutant embryos. Mech Dev 2002, 117:115-122

36. Novak A, Guo C, Yang W, Nagy A, Lobe CG: Z/Eg, a double reporter mouse line that expresses enhanced green fluorescent protein upon cre-mediated excision. Genesis 2000, 28:147-155.

37. Maden CH, Gomes J, Schwarz Q, Davidson K, Tinker A, Ruhrberg C: Nrp1 and Nrp2 cooperate to regulate gangliogenesis, axon guidance and target innervation in the sympathetic nervous system. Dev Biol 2012, 369:277-285.

38. Ma Q, Fode C, Guillemot F, Anderson DJ: Neurogenin1 and neurogenin2 control two distinct waves of neurogenesis in developing dorsal root ganglia. Genes Dev 1999, 13:1717-1728.

39. Lee EC, Yu D, Martinez De Velasco J, Tessarollo L, Swing DA, Court DL, Jenkins NA, Copeland NG: A highly efficient Escherichia coli-based chromosome engineering system adapted for recombinogenic targeting and subcloning of Bac dna. Genomics 2001, 73:56-65.

40. Liu P, Jenkins NA, Copeland NG: A highly efficient recombineering-based method for generating conditional knockout mutations. Genome Res 2003, 13:476-484

41. Wiszniak S, Kabbara S, Lumb R, Scherer M, Secker G, Harvey N, Kumar S, Schwarz Q: The ubiquitin ligase nedd4 regulates craniofacial development by promoting cranial neural crest cell survival and stem-cell like properties. Dev Biol 2013, 383:186-200.

42. Schwarz Q, Gu C, Fujisawa H, Sabelko K, Gertsenstein M, Nagy A, Taniguchi M, Kolodkin AL, Ginty DD, Shima DT, Ruhrberg C: Vascular endothelial growth factor controls neuronal migration and cooperates with SEMA3a to pattern distinct compartments of the facial nerve. Genes Dev 2004, 18:2822-2834

43. Singh S, Bhattacherjee V, Mukhopadhyay P, Worth CA, Wellhausen SR, Warner CP, Greene RM, Pisano MM: Fluorescence-activated cell sorting of eGFP-labeled neural crest cells from murine embryonic craniofacial tissue. J Biomed Biotechnol 2005, 2005:232-237.

doi:10.1186/1749-8104-9-24

Cite this article as: Lumb et al:: Neuropilins define distinct populations of neural crest cells. Neural Development 2014 9:24.

\section{Submit your next manuscript to BioMed Central and take full advantage of:}

- Convenient online submission

- Thorough peer review

- No space constraints or color figure charges

- Immediate publication on acceptance

- Inclusion in PubMed, CAS, Scopus and Google Scholar

- Research which is freely available for redistribution 